\title{
Predicting Impact of COVID-19 on Crude Oil Price Image with Directed Acyclic Graph Deep Convolutional Neural Network
}

\author{
David Opeoluwa Oyewola $^{1 *}$, Akomolafe Femi Augustine ${ }^{2}$, Emmanuel Gbenga Dada ${ }^{3}$, Asabe Ibrahim ${ }^{1}$ \\ ${ }^{1,1}$ Department of Mathematics \& Computer Science, Federal University of Kashere, Gombe. \\ ${ }^{2}$ Department of Economics and Development Studies, Federal University of Kashere, Gombe. \\ ${ }^{3}$ Department of Mathematical Sciences, University of Maiduguri, Maiduguri \\ Email: ${ }^{1}$ davidakaprof01@yahoo.com, ${ }^{2}$ austinesq@yahoo.com, ${ }^{1}$ asabeibrahim@gmail.com, \\ ${ }^{3}$ gbengadada@unimaid.edu.ng \\ *Corresponding Author
}

\begin{abstract}
In sequential data, prediction and image classification, deep learning methods have obtained outstanding results. In this study, we propose image transformation of time series crude oil price by incorporating Directed Acyclic Graph to Convolutional Neural Network (DAG) based on image processing characteristics. Crude oil price time series is converted into 2-D images, utilizing 10 distinctive technical indicators. Geometric Brownian Motion was utilized to produces data for a 10-day time span. Thus, a 2-D image with a size of 10x10 sized 2-D is constructed. Then mark each image as "buy" or "sell" based on time series returns. The results show that integrating DAG with CNN improves the prediction accuracy by $14.18 \%$. DAG perform best with an accuracy of $99.16 \%$, sensitivity of $100 \%$ and specificity of $99.19 \%$. COVID19 has negatively affected Nigeria crude oil price which indicates a downward trend of crude oil price. The study recommends poly-cultural economy of Nigeria economy for national development of the nation.
\end{abstract}

Keywords-COVID-19, Recurrent Neural Network, Convolution Neural Network, Directed Acyclic Graph, Long Short Term Memory, Geometric Brownian Motion.

\section{INTRODUCTION}

The first case of Coronavirus was reported in Wuhan Jinyintan Hospital on 30 December 2019 from a patient with obscure etiology. As of 14 May 2020, coronavirus pandemic had spread to 213 countries with 4,464,255 cases, 299,410 deaths and 1,678,246 recovered cases [2]. Coronavirus coupled with a price war between Saudi Arabia and Russia, have catastrophically affected Nigerian crude oil price. The survival of Nigeria economy greatly relies on the crude oil price. The costs of oil have drastically reduced by half since January 2020, with the USA crude market record exceptionally low [1]. Numerous projects have greatly affected the drooped in the oil cost. The slump in oil price has impacted and adversely affected the Nigerian economy [3]. The investigation among others suggests that broadening of the Nigerian economy is most basic given the financial downturn in the nation now. The author [4] argued that Nigeria remains helpless to the developments in global unrefined petroleum costs as a mono-product economy. Oil is essential to financial and money related strategies in Nigeria since it represents for $80 \%$ of government income, $90-95 \%$ of foreign exchange profits and $12 \%$ of the real gross domestic product [5]. Recently, there has been a huge enthusiasm for deep learning [6]. Deep learning algorithms have produced outstanding results in time series, medical research, natural language processing, image classification and computer vision [7]. By incorporating two convolutional neural networks (CNN) with various structures, the authors [8] proposes Dual-Channel Convolution Neural Network (DCCNN) model. The results show that DCCNN achieves a labelling accuracy higher than that of Convolution Neural Network (CNN). As indicated by [9] proposed AlexNet model which showed a huge improvement in the image classification task.

\section{METHODOLOGY}

\section{A. Data Set}

The dataset used in this paper comprises of COVID-19 data obtained from Kaggle database. The data is made up of data from $22 / 1 / 2020$ to $23 / 3 / 2020$ and daily crude oil price in Nigeria was extracted from www.cbn.gov.ng. Crude oil prices were recorded at the end of every day from 01/02/2019 to $03 / 23 / 2020$. In this study, crude oil price information for that period was taken for analysis using time series. The returns are calculated using (1).

The return is as follows:

$r_{t}=\frac{C(t+\Delta t)-C(t)}{C(t)}$ 
where $r_{t}$ is the crude oil price return, $C(t)$ is the closing crude oil price at time $t$ and $\Delta C(t)$ is the small change in crude oil closing price at time $t$.

\section{B. Geometric Brownian Motion}

Geometric Brownian Motion is a stochastic process that follows a Brownian motion or wiener process with drift [29].

The Geometric Brownian Motion is:

$c_{t}=c_{o} \exp ^{\left(\mu t+\sigma W_{t}\right)}$

Where $c_{o}$ is the initial crude oil price $c_{t}$ closing crude oil price at time $t, \mu$ is the mean, $\sigma$ is the variance, $W_{t}$ is the wiener process at time $t$.

C Auto Encoder

Autoencoder is unsupervised learning that comprises three layers: a hidden layer, output layer, input layer as shown in Fig. 1. Two important elements which are encoder and decoder components are involved in an autoencoder. An encoder maps the input image to the hidden image, while the decoder map returns this hidden image to the original input image. The input dataset $\left\{x_{n}\right\}$ is the $10 \times 10$ matrices obtained from the ten technical indicators used in this research.

The encoding and decoding process is as follows:

$h_{n}=\sigma\left(W_{1} x_{n}+b_{1}\right)$

$\hat{x}_{n}=\rho\left(W_{2} h_{n}+b_{2}\right)$

where $\sigma$ is the encoding function, $\rho$ is the decoding Function, $W_{1}$ is the weight matrix of the encoder, $W_{2}$ is the weight matrix of the decoder, $b_{1}$ is the bias vector of the encoder, $b_{2}$ is the bias vector of the decoder.

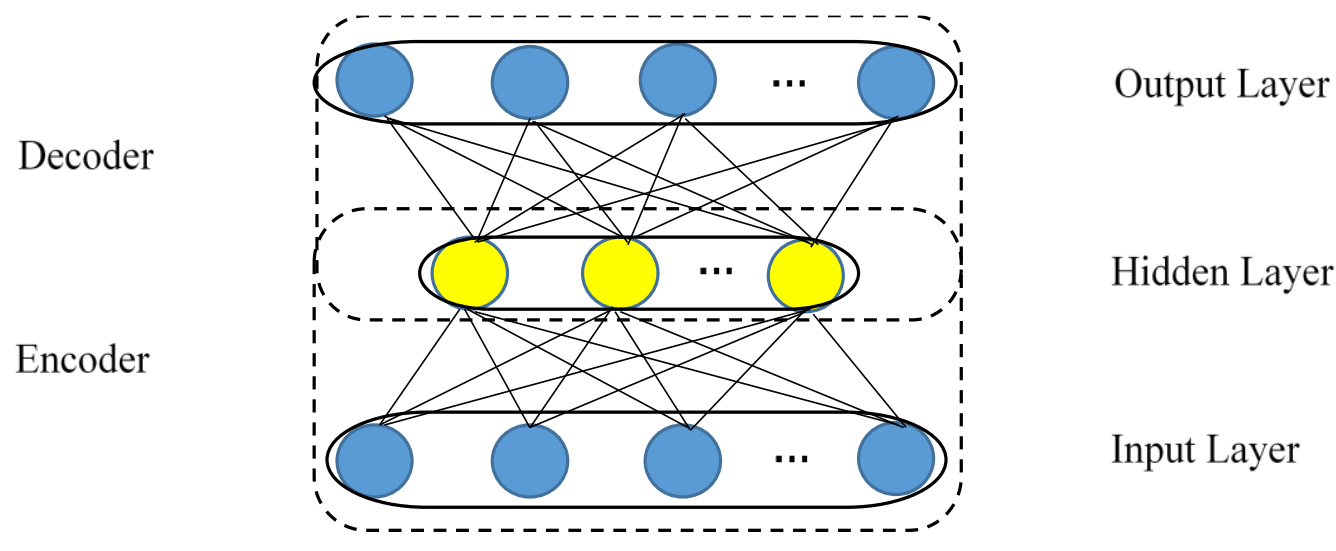

Fig. 1. Structure of Autoencoder

\section{Technical Indicators}

In this study, ten technical indicators will be considered which are SMA, RSI, CMO, DPO, ROC, EMA, WMA, ZLEMA, DEMA and HMA are selected as $10 \times 10$ image creation for the proposed model.

The technical indicators are calculated from Geometric Brownian Motion (GBM) as follows:

- $\quad$ Relative Strength Index (RSI)

The RSI formula is as follows:

$R S I=100-\frac{100}{1+\left(\sum_{i=0}^{n-1} \frac{U p_{t-i}}{n}\right) /\left(\sum_{i=0}^{n-1} D w_{t-i} / n\right)}$

- $\quad$ Chande Momentum Oscillator (CMO)

The CMO formula is given as:

$C M O=100 \times \frac{\sum_{i=0}^{n-1} U p_{t-i}-\sum_{i=0}^{n-1} D w_{t-i}}{\sum_{i=0}^{n-1} U p_{t-i}+\sum_{i=0}^{n-1} D w_{t-i}}$

- $\quad$ Detrended Price Oscillator (DPO)

$\mathrm{DPO}$ is given as:

$D P O=C_{t}-\operatorname{SMA}\left(\frac{n}{2}+1\right)$
- $\quad$ Rate of Change (ROC)

ROC formula measure as:

$R O C_{t}=\frac{C_{t}-C_{t-n}}{C_{t-n}}$

- $\quad$ Exponential Moving Average (EMA)

Exponential moving average of $n$ days is calculated as:

$E M A_{t}=C_{t} \times \propto+E M A_{t-1} \times(1-\propto)$

$\propto=2 \div(n+1)$

- $\quad$ Simple Moving Average (SMA)

Simple Moving Average is measured as:

$S M A_{t}=\frac{1}{t} \sum_{i=1}^{t} C_{t}$

- Weighted Moving Average (WMA)

WMA is calculated as:

$W M A=\left(C_{t} \times n+C_{t-1} \times(n-1)+\cdots C_{t}(n-1) \times 1\right) /$

$\left(\frac{n(n+1)}{2}\right)$

- Zero Lag Exponential Moving Average (ZLEMA) ZLEMA is as follows: 
$E M A 1=\left[C_{t}+\left(C_{t}-C_{t}\left(\frac{n-1}{2}\right)\right)\right]$

$Z L E M A=E M A(E M A 1, n)$

- $\quad$ Double Exponential Moving Average (DEMA) DEMA is defined as:

$D E M A=2 \times E M A(n)-E M A(E M A(n))$

- Hull Moving Average (HMA)

HMA is measured as:

$\left.H M A=W M A\left(2 \times W M A\left(\frac{n}{2}\right)-W M A(n)\right), \sqrt{n}\right)$

Where $U p_{t}$ is the upward-price-change, $D w_{t}$ is the downward-price-change at time $t, C_{t}$ is the closing price at time $t, S M A$ the simple moving average, $n$ is time period and $\propto$ is the exponential smoothing.

\section{E. Labelling}

In this research, a technique that utilizes a Convolution Neural Network to predict the image transformation of daily crude oil prices is proposed. Ten Technical indicators which include SMA, RSI, CMO, DPO, ROC, EMA, WMA, ZLEMA, DEMA and HMA is for creating images. We utilize Geometric Brownian Motion (GBM) to extract daily crude oil price of each day for the period of 10 days' period. This study aims primarily to identify the best prediction models for the price of crude oil.

Label play a significant role in image classification. The extracted data in 2.1 was labelled using equation (1) in marking the daily crude oil prices as "Buy" or "Sell" this helps us in determining the up and down signals. An upward pattern is signaled by a positive return while descending pattern is signaled by a negative return. In the event the return is positive, it is labelled "Buy" and if the direction return is negative, it is labelled "Sell".

\section{F. Image Creation}

Geometric Brownian Motion (GBM) model which is being used in mathematical finance. The crude oil price, we presume, follows a random walk and that prices are integrated and the next price increases is based on previous price adjustment. Geometric Brownian Motion in [29] equation (2) was utilized in the first phase of image creation which enables us to runs 10-day period of crude oil price obtained from each daily crude oil price. The initial value $c_{o}$ is represented as daily crude oil closing price, mean and variance is obtained from the crude oil closing price as demonstrated in table I. The second phase of the image creation was obtained from the ten-day trials of each of the GBM in which ten technical indicators was utilized. The technical indicator utilized in this paper are SMA, RSI, CMO, DPO, ROC, EMA, WMA, ZLEMA, DEMA and HMA values are determined using $\mathrm{R}$ programming library at different intervals of 2 days. Each day the $10 \times 10$ matrices were generated from the 10 technical indicators. Third stage is to extract the feature of all the $10 \times$ 10 matrices using Autoencoder. For each day a 10 x 10 pixels image with an autoencoder is produced and resized to $30 \times 30$ pixels. In the meantime, the approach given in section 2.2 applies to each image using the corresponding mark ("Buy" or "Sell") image. Fig. 2 illustrates a sample of 30 x 30-pixel images generated during the development process of the image. In our study, the closing data for crude oil prices are estimated at around 254 images in a 1 year and 3-months.

TABLE I. TEN-DAY TRIALS OF GBM

\begin{tabular}{|c|c|c|}
\hline Date & Price & GBM \\
\hline \multirow[t]{10}{*}{ 01-02-19 } & 52.63 & 52.63 \\
\hline & & 51.68356 \\
\hline & & 49.46833 \\
\hline & & 46.66414 \\
\hline & & 49.04746 \\
\hline & & 49.46757 \\
\hline & & 51.37479 \\
\hline & & 52.65546 \\
\hline & & 55.13887 \\
\hline & & 57.86565 \\
\hline \multirow[t]{10}{*}{ 01-03-19 } & 54.75 & 54.75 \\
\hline & & 56.11687 \\
\hline & & 55.10772 \\
\hline & & 52.74573 \\
\hline & & 49.75575 \\
\hline & & 52.29698 \\
\hline & & 52.74492 \\
\hline & & 54.77849 \\
\hline & & 56.14401 \\
\hline & & 58.79196 \\
\hline
\end{tabular}

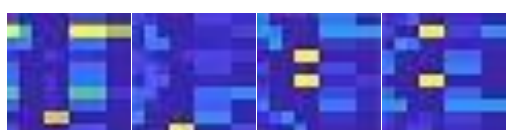

Fig. 2. Image 30 x 30 Pixel Sample Images

\section{G. Proposed Convolution Neural Network}

In both regression and classification, Convolution Neural Network can be utilized. Our proposed method uses the CNN for both regression and classification of crude oil price. In order to utilize the regression capability of $\mathrm{CNN}, 18$ layers are utilized which include: image input layer $(30 \times 30)$, four convolutional layer, two average pooling layer, four batch normalization layer, four rectified linear unit, one dropout layer, one fully connected layer and an output regression layer. Batch normalization layer is added to improve the $\mathrm{CNN}$ 
stability of the input data (as shown in Fig. 3). Classification of "Buy" and "Sell" utilized in this paper comprises of the image input layer $(30 \times 30)$, three convolution layer, three batch normalization layer, three rectified linear unit, two max-pooling layers, two fully connected layers, SoftMax layer and a classification layer (as shown in Figure 4).

Directed Acyclic Graph (DAG) is a graph of cycles, which have coordinated paths from start and end in a similar node. It is a diagram without any cycles. In this paper, we utilize DAG by integrating it with CNN features. Fig. 5 shows
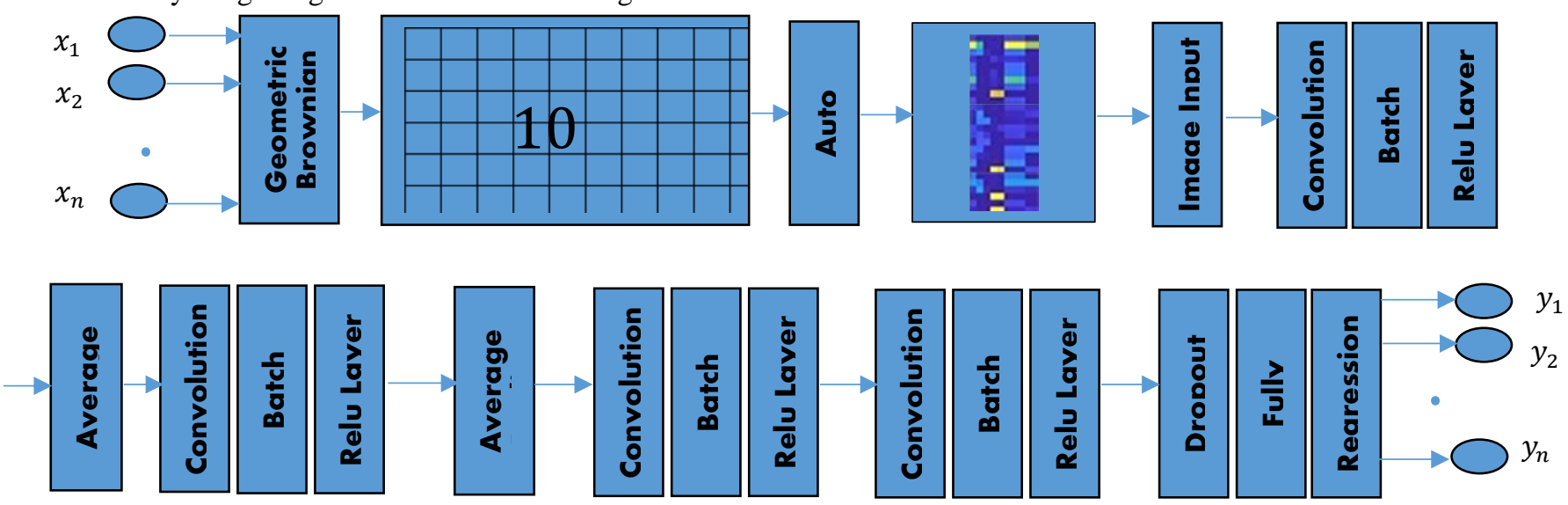

Fig. 3: Structure of Sequential Convolution Neural Network
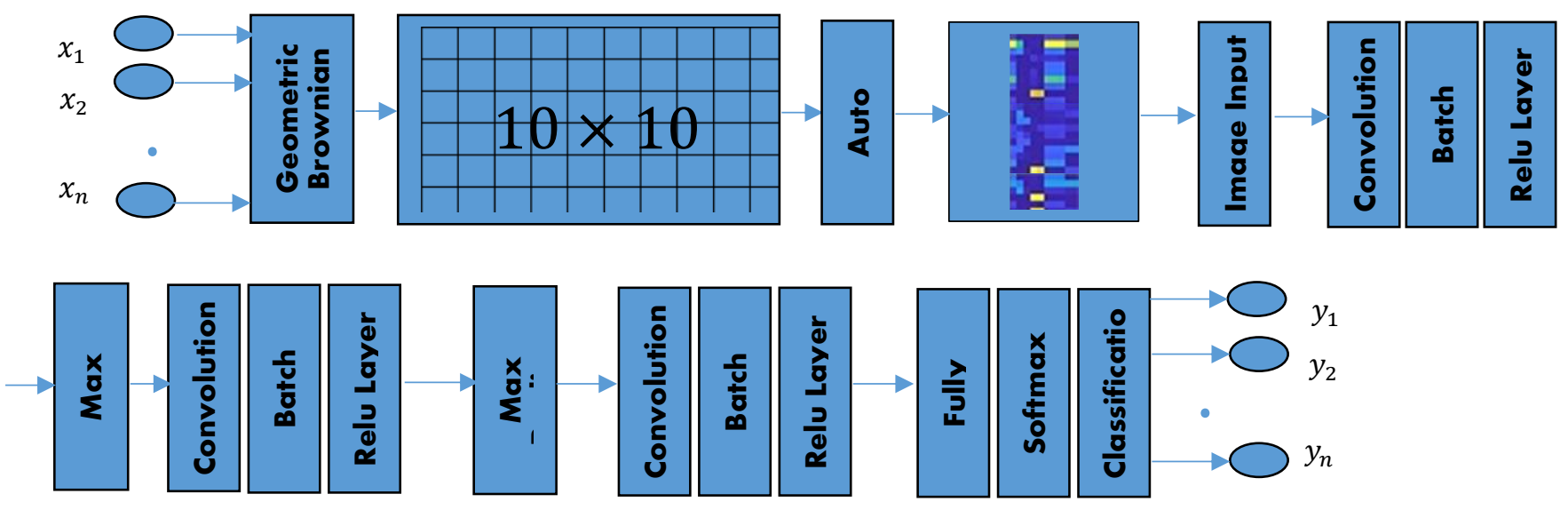

Fig. 4: Structure of Classification Convolution Neural Network

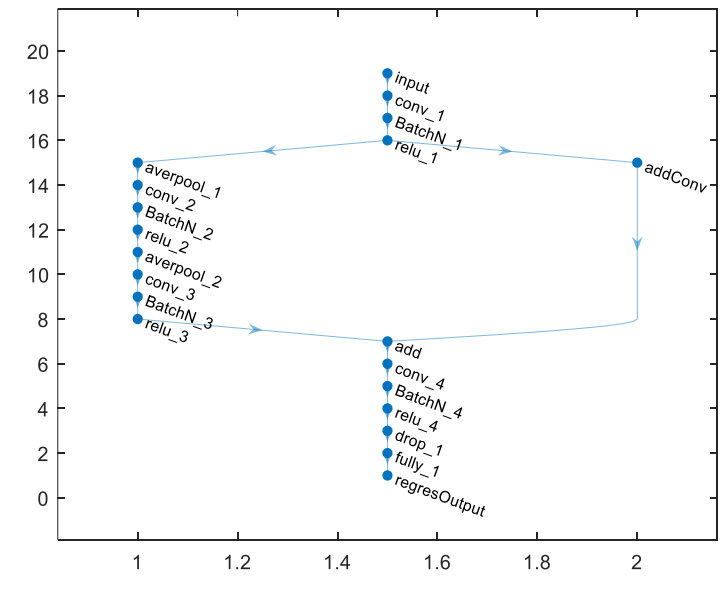

Fig. 5. Structure of Regression Directed Acyclic Graph Convolution Neural Network

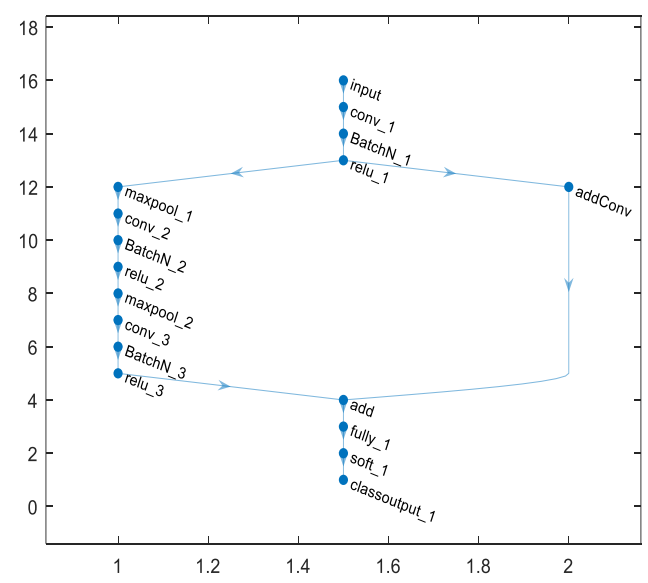

Fig. 6. Structure of Classification Directed Acyclic Graph Convolution Neural Network 


\section{III.RESULT AND DISCUSSION}

We compared four deep learning techniques. Two multilayer analyzes such as Convolution Neural Network (CNN) and Directed Acyclic Graph with the integration of CNN, whereas the other two are also multi-layer network, used for sequence analysis of inputs such as Recurrent Neural Network (RNN) and Long Short-Term Memory (LSTM). Overall performance of Convolution Neural Network (CNN) model is assessed by Mean Absolute Error (MAE), Root Mean Square Error (RMSE) and Mean Absolute Scaled Error (MASE).

Table II shows the performance evaluation of each criterion utilizes in this paper. As indicated by Table II and Fig. 7, RNN has the lowest error compared to other algorithms.

However, the performance of CNN, RNN and LSTM classification model is evaluated using overall accuracy, sensitivity and specificity (as shown in Table III-VII) to illustrate how precisely the classifier distinguishes between Buy, and Sell. The CNN, DAG, RNN and LSTM of crude oil prices classification are shown in Table III-VI. Table VII displays the comparison of accuracy, sensitivity and specificity criteria. Table VII in contrast to other deep learning algorithms CNN failed to achieve the best result when compared to other deep learning algorithms. DAG performs best with $99.61 \%, 100 \%$ and $99.19 \%$ respectively, followed by RNN and CNN.

Crude Oil prices play a significant role in the Nigeria economy growth due to immensely dependence on the oil prices for economic survival. Fig. 8 displays crude oil price time series and COVID-19 cases from January to March 2020. The crude oil price was stable from January to February which indicates that COVID-19 has no effect on the price of crude oil. With the increase of COVID-19 cases rising in February, COVID-19 sparked a downward trend of crude oil price. It indicates that the COVID-19 had a negative impact on the economy of Nigeria between February and March.

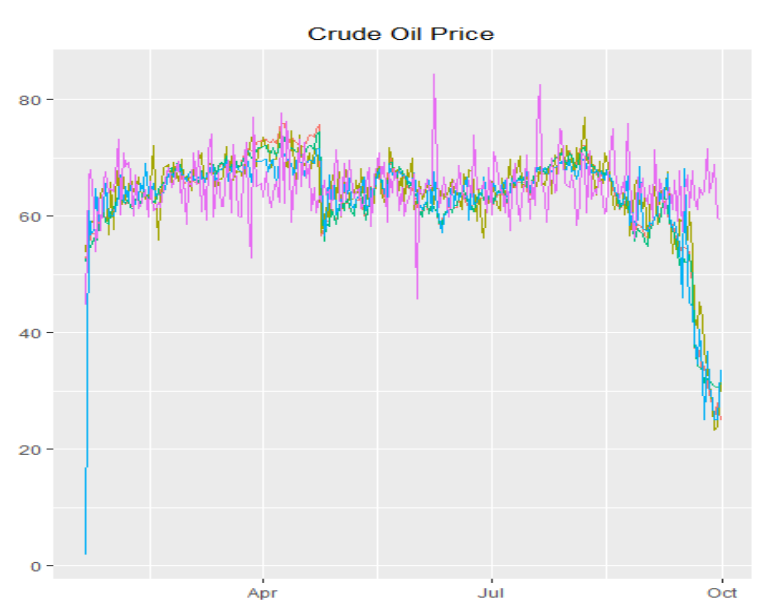

Fig. 7. Crude oil Price Prediction

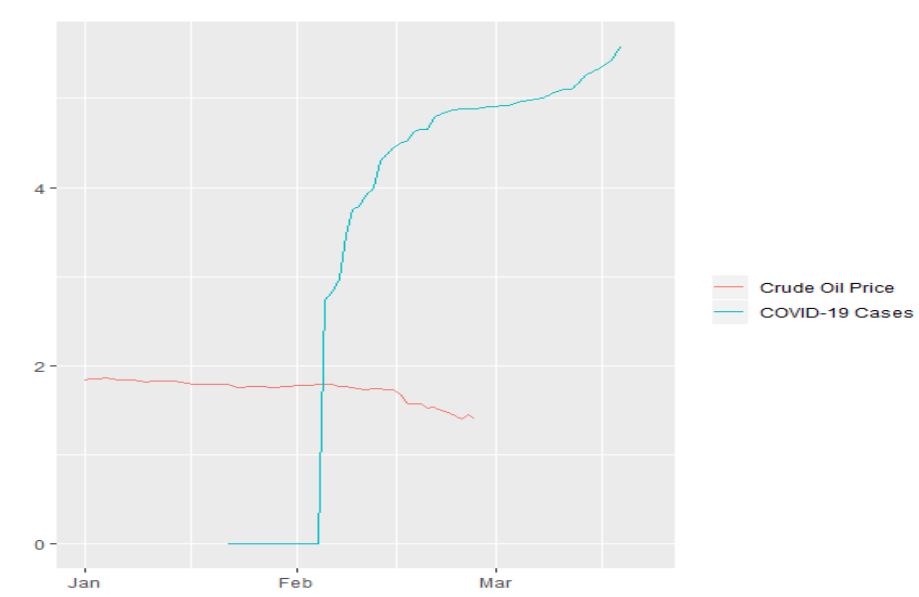

Fig. 8. Time Series of Crude oil price and Covid-19 Cases

TABle II. Performance Evaluation of Regression of CNN, DAG, RNN AND LSTM

\begin{tabular}{|l|l|l|l|}
\hline MODEL & MAE & RMSE & MASE \\
\hline CNN & 2.8416 & 3.4704 & 1.2078 \\
\hline DAG & 6.1935 & 9.2961 & 2.6325 \\
\hline RNN & 0.7952 & 1.2542 & 0.3380 \\
\hline LSTM & 2.2030 & 4.3808 & 0.9364 \\
\hline
\end{tabular}

TABLE III. CONFUSION MATRIX OF CNN

\begin{tabular}{|l|l|l|}
\hline CNN & BUY & SELL \\
\hline BUY & 115 & 21 \\
\hline SELL & 16 & 102 \\
\hline
\end{tabular}

TABLE IV. CONFUSION MATRIX OF LSTM

\begin{tabular}{|l|l|l|}
\hline LSTM & BUY & SELL \\
\hline BUY & 91 & 53 \\
\hline SELL & 40 & 70 \\
\hline
\end{tabular}

TABLE V. CONFUSION MATRIX OF RNN

\begin{tabular}{|l|l|l|}
\hline RNN & BUY & SELL \\
\hline BUY & 115 & 10 \\
\hline SELL & 16 & 113 \\
\hline
\end{tabular}

TABLE VI. CONFUSION MATRIX OF DAG

\begin{tabular}{|l|l|l|}
\hline DAG & BUY & SELL \\
\hline BUY & 131 & 1 \\
\hline SELL & 0 & 122 \\
\hline
\end{tabular}

TABLE VII: PERformance EVAluATION ClassifiCATION OF CNN, DAG, LSTM AND RNN

\begin{tabular}{|l|l|l|l|}
\hline MODEL & \multicolumn{1}{|c|}{$\begin{array}{c}\text { Accuracy } \\
(\boldsymbol{\%})\end{array}$} & $\begin{array}{c}\text { Sensitivity } \\
(\boldsymbol{\%})\end{array}$ & $\begin{array}{c}\text { Specificity } \\
(\boldsymbol{\%})\end{array}$ \\
\hline CNN & 85.43 & 87.79 & 82.93 \\
\hline DAG & 99.61 & 100 & 99.19 \\
\hline LSTM & 63.39 & 69.47 & 56.91 \\
\hline RNN & 89.76 & 87.79 & 91.87 \\
\hline
\end{tabular}

\section{CONCLUSION}

Deep learning has achieved surprising accomplishments over a variety of domains that include classification and prediction, image classification, object detection, sequential data such as text, speech or videos. We leverage the 
advantage of CNN and DAG to predict the prices and effect of crude oil price on COVID-19. The result showed that price of crude oil can be transformed into images for both prediction and classification. Likewise, due to COVID-19 pandemic, the downturn in oil prices has a negative effect on Nigerian economy because of the huge dependence on the crude oil price. The study recommends poly-cultural economy of Nigeria economy for national development of the country.

\section{REFERENCE}

[1] https://www.offshore-technology.com/investment/covid19-creates-supply-and-demand- crisis-for-oil-andgas/ (Accessed on 14 May 2020)

[2] https://www.worldometers.info/coronavirus/ (Accessed on 14 May 2020)

[3] Nwoba, M. O, Nwonu, C. and Agbaeze, E.K, Impact of Fallen Oil Prices on the Nigeria Economy, Journal of Poverty, Investment and Development, 2017, vol. 33, pp. $75-82$

[4] Ayoola, J, "Does Volatility in Crude Oil Price Precipitate Macroeconomic Performance in Nigeria?" International Journal of Energy Economics Policy. 3 (2) 143152,2013

[5] Yusuf, M, "An Analysis of the Impact of Oil Price Shocks on the Growth of the Nigerian Economy", 1970-2011. African Journal of Business Management, 2015, 9(3), 103-115.

[6] LeCun Y, Bengio Y, Hinton G, Deep learning. Nature 521: 436-444, 2015

[7] CiresAn, D., Meier, U., Masci, J., and Schmidhuber, J., "Multi-column deep neural network for traffic classification". Neural Network, 2012, 32,333-338.

[8] Jianfang Cao, Chenyan Wu, Lichao Chen, Hongyan Cui, and Guoqing Feng, "An Improved Convolutional Neural Network Algorithm and Its Application in Multilabel Image Labeling”, Computational Intelligence and Neuroscience 2019, Volume 1, pp. 111.

[9] Alex Krizhevsky, Ilya Sutskever, and Geoffrey E Hinton, Imagenet classification with deep convolutional neural networks,"in Advances in neural information processing systems, 2012, pp. 1097-1105.

[10] Alex Graves, Abdel-rahman Mohamed, and Geoffrey Hinton, Speech recognition with deep recurrent neural networks, in Acoustics, speech and signal processing (icassp), ieee international conference on. IEEE, 2013, pp.6645-6649.

[11] Yushi Chen, Hanlu Jiang, Chunyang Li, Xiuping Jia, and Pedram Ghamisi, "Deep feature extraction and classification of hyperspectral images based on convolutional neural networks", IEEE Trans. Geosci. Remote Sens., 2016, vol. 54, no. 10, pp. 6232-6251.

[12] S. Zhu, S. Shen, and X. Li, "Multimodal deep network learning-based image annotation", Electronics Letters, 2015, vol. 51, no. 12, pp. 905-906.

[13] J. Deng, W. Dong, R. Socher, L. J. Li, K. Li, and F. F. Li, "Imagenet: a large-scale hierarchical image database", in Proceedings of the IEEE Conference on
Computer Vision and Pattern Recognition (CVPR 2009), 2009, pp. 248-250, Miami, FL, USA.

[14] L. X. Jaing and J. Hou, Image annotation using the ensemble learning, Acta Autamatic Sinica, 2012, vol. 38 , no. 8, pp. 1257-1262.

[15] C.C. Aggarwal, Neural Networks and Deep Learning- A Textbook, Springer, 2018.

[16] D. Ciresan, U. Meier, and J. Schmidhuber, "Multicolumn deep neural networks for image classification", 2012, DOI: 10.1109/CVPR.2012.6248110.

[17] Simonyan, Karen, and Andrew Zisserman, "Very deep convolutional networks for large-scale image recognition", 2014, arXiv preprint arXiv:1409-1556.

[18] Kim, Ho-Joon, Joseph S. Lee, and Hyun-Seung Yang, Human action recognition using a modified convolutional neural network. International Symposium on Neural Networks. Springer Berlin Heidelberg, 2007.

[19] Jonathan, A Weyn, Dale, R Durran, Rich Caruana, "Improving Data-Driven Global Weather Prediction Using Deep Convolutional Neural Networks On a Cubed Sphere", arXiv:2003.1927v1[physics.ao-ph] 15 Mar 2020, pp. 1-15.

[20] W. Boomsma and J. Frellsen, "Spherical convolutions and their application in molecular modelling". In Adv.Neural Inf. Process. Syst., 2017, volume 4, pages 3434-3444.

[21] A. Chattopadhyay, E. Nabizadeh, and P. Hassanzadeh, "Analog forecasting of extreme-causing weather patterns using deep learning". J. Adv. Model. Earth Syst., vol. 5, pp. 1-5.

[22] G. R. Herman and R. S. Schumacher, “Money Doesn't Grow on Trees, But Forecasts Do: Forecasting Extreme Precipitation with Random Forests". Weather Rev., 2020, 146(5):1571-1600.

[23] P. Golik, Z. Tuske, R. Schl ". uter, and H. Ney, "Convolutional neural networks for acoustic modeling of raw time signal in LVCSR", in Proc. Annual Conference of International Speech Communication Association (INTERSPEECH), 2015.

[24] P. Swietojanski, A. Ghoshal, and S. Renals, "Convolutional neural networks for distant speech recognition," IEEE Signal Processing Letters, 2014, vol. 21, no. 9, pp. 1120-1124.

[25] Anthimopoulos M, Christodoulidis S, Ebner L, et al. "Lung pattern classification for interstitial lung diseases using a deep convolutional neural network". IEEE Trans Med Imaging. 2016, 35:1207-16.

[26] Ker J, Wang L, Rao J, Lim T. "Deep learning applications in medical image analysis". IEEE Access. 2018;6:9375-89.

[27] Kenig, T., Kam, Z., Feuer, A. "Blind image deconvolution using machine learning for three dimensional microscopy". IEEE Trans. Pattern Anal. Mach. Intell, 2010, 32(12), pp. 23-30.

[28] S. Scher. "Toward Data-Driven Weather and Climate Forecasting: Approximating a Simple General Circulation Model with Deep Learning”. Geophys. Res. Lett., 2018, 45(22):12, 616-622.

[29] David O. Oyewola, Aye Patrick Olabanji, Terrang.A.U, Jayeola Dare, "Deep Continuous-Time Models in 
Nigerian Stock Exchange Sector", 2020, Journal of Science and Technology Research 2(1) pp. 99-113

[30] David Oyewola, Danladi Hakimi, Kayode Adeboye, Musa Danjuma Shehu, "Using Five Machine Learning for Breast Cancer BiopsyPredictions Based on Mammographic Diagnosis", International Journal of Engineering Technologies-IJET, 2016, Vol.2, No.4, pp. $142-14$ 